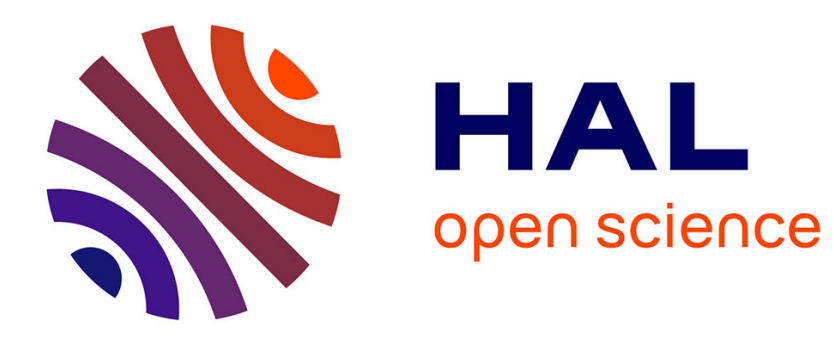

\title{
A unified perturbation approach to static/dynamic coupled instabilities of nonlinear structures
}

\author{
Angelo Luongo
}

\section{To cite this version:}

Angelo Luongo. A unified perturbation approach to static/dynamic coupled instabilities of nonlinear structures. Thin-Walled Structures, 2010, 48 (10-11), pp.744-751. hal-00788398

\section{HAL Id: hal-00788398 \\ https://hal.science/hal-00788398}

Submitted on 14 Feb 2013

HAL is a multi-disciplinary open access archive for the deposit and dissemination of scientific research documents, whether they are published or not. The documents may come from teaching and research institutions in France or abroad, or from public or private research centers.
L'archive ouverte pluridisciplinaire HAL, est destinée au dépôt et à la diffusion de documents scientifiques de niveau recherche, publiés ou non, émanant des établissements d'enseignement et de recherche français ou étrangers, des laboratoires publics ou privés. 


\title{
A unified perturbation approach to static/dynamic coupled instabilities of nonlinear structures
}

\author{
A. Luongo* \\ DISAT, University of L'Aquila, Monteluco Roio, 67040 L'Aquila, Italy
}

\begin{abstract}
A B S T R A C T
A unified perturbation approach, based on the multiple scale method, able to analyse interactions among static and/or dynamic bifurcations of nonlinear structures, is illustrated. The method appears as the natural extension to the dynamical case of the static perturbation method, well known in buckling of elastic structures. To illustrate the algorithm, a general, multiparameter, finite dimensional autonomous system is considered, simultaneously undergoing a static (divergence) and two dynamic (Hopf) bifurcations, of nonresonant or resonant type. The method furnishes bifurcation equations governing the time evolution of amplitudes and phases of the critical interacting modes. Results relevant to sample structures are discussed.
\end{abstract}

\section{Introduction}

Coupled instability phenomena, often referred to as 'multiple' or 'simultaneous buckling mode interaction', often occur in statics of nonlinear elastic structures. They are well known to research ers and practitioners and have been object of extended studies in the last few decades [ $\left.\begin{array}{ll}1 & 5\end{array}\right]$.

A coupled static instability phenomenon manifests itself, along a 'known' path $\Gamma_{0}$ of a load displacement space, when, at a bifurcation point $C$, the tangent stiffness matrix admits an eigenvalue $A=0$ of multiplicity $M \geq 2$. As a result, more than one path $\Gamma_{j}$ bifurcate from $\Gamma_{0}$, each corresponding to different buckling patterns, which, in the neighbourhood of $C$, are close to linear combinations of the $M$ critical modes. It is also well known that one or more splitting (or detuning) parameters $\rho_{h}$ (typically, geometric aspect ratios) must be adjusted, in order that a multiple bifurcation occurs. A paradigmatic example of such a behaviour is offered by the famous 2 d.o.f. Augusti model (Fig. 1a), for which four bifurcated paths exist for $M=2$, when the stiffness ratio is $\rho=1$ (Fig. 1b). If the splitting parameters are not exactly tuned, 'nearly simultaneous' buckling modes occur, entailing more critical point $C_{i}$ clustered on $\Gamma_{0}$, close to other 'secondary' bifurcation points $C_{j}$ belonging to $\Gamma_{j}(i=1,2$ and $j=3,4$ in the example of Fig. 1c).

A simple and efficient method to deal with such problems is offered by the static perturbation method (SPM) (see, e.g. [6 8 8]). It consists: (1) in expanding the displacement variables $\mathbf{w}$ and the load parameter $\mu$ in series of an artificially introduced

\footnotetext{
* Tel.: +390862434521; fax: +390862434548.

E-mail addresses: luongo@ing.univaq.it, angelo.luongo@univaq.it.
}

perturbation parameter $\varepsilon \ll 1$; $(2)$ in solving a sequence of linear perturbation equations in the unknown coefficients $\mathbf{w}_{k}$ and $\mu_{k}$ of the series expansions; and, (3) in expressing the bifurcated paths in parametric form, $\mathbf{w}=\mathbf{w}(\varepsilon), \mu=\mu(\varepsilon)$. In this perturbation procedure, a key role is played by the solvability (or compat ibility) condition' (namely, the bilinear identity of linear algebra, or the Fredholm alternative of functional analysis), i.e. the condition under which a linear problem with singular operator admits solution. Such a condition requires the known terms of each perturbation equation are orthogonal to all the critical modes $\mathbf{u}_{k}$, and supplies, at any order, the set of nonlinear equations linking the coefficients of the linear combinations of $\mathbf{u}_{k}$ (i.e. the 'amplitudes' of the buckling modes) and $\mu_{k}$. It is worth noticing that the SPM works as a reduction method, since it reduces the (generally large) dimension of the problem to the (generally small) dimension $M$.

Several assumptions are understood in the elastic buckling analysis described above, namely:

(1) The load $\mu$ is taken as unique control parameter, and splitting parameters $\rho_{h}$ are adjusted to the values causing coalescence of $M$ eigenvalues $A$, and (usually) kept constant. The influence of the splitting parameters is investigated by repeating the analysis for slightly modified values, considered as imperfections.

(2) The system is conservative, i.e. the stiffness matrix is symmetric. This entails a complete set of eigenvectors exist, and right and left eigenvectors coincide.

(3) Nonconservative forces are excluded, so that bifurcations are of static type only. (Flutter instability, as e.g. induced by follower forces, is analysed outside this context, by methods often borrowed from nonlinear dynamics). 

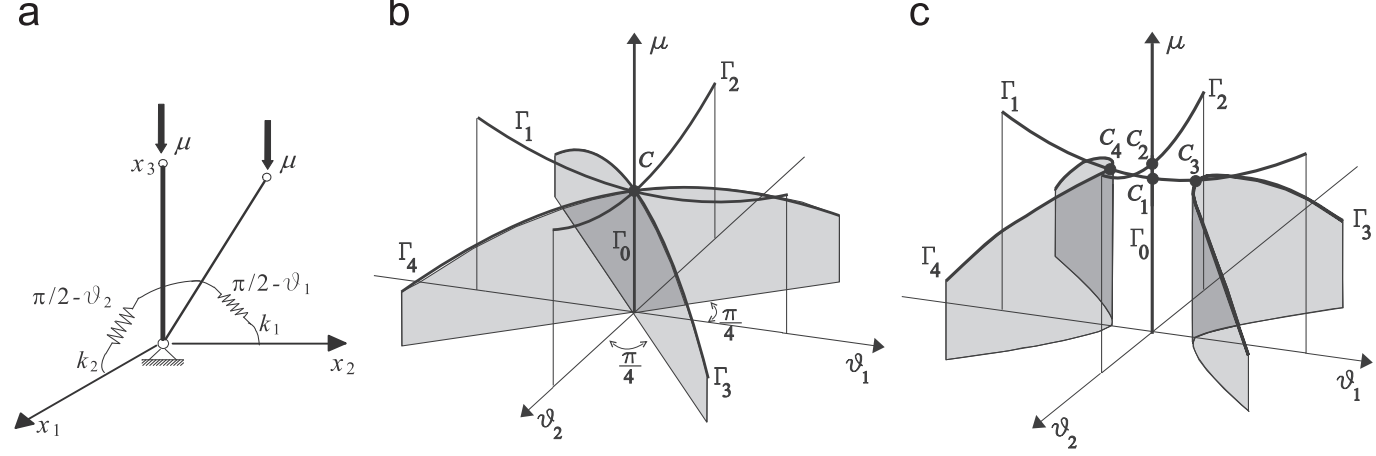

Fig. 1. (a) Augusti model $\left(\rho:=k_{2} / k_{1}\right)$, (b) paths for simultaneous modes $(\rho=1)$ and (c) paths for nearly simultaneous modes $(\rho \simeq 1)$.

One, of course, could be interested in removing one or more of the previous hypotheses. In particular, to deal with loads depending on several independent parameters, and/or families of systems (not just a special system!) described by the splitting parameters. These aspects call for considering multiparameter systems, for which the concept of 'equilibrium paths' must be generalized to 'equilibrium surfaces'. Moreover, damping and nonconservative forces should be included in the analysis, in order to study dynamic bifurcations. As a consequence, the configuration space must be enlarged to the state space (including velocities), and the tangent (Jacobian) matrix $\mathbf{F}_{\mathbf{x}}^{0}$ considered (accounting for inertial, dissipative, potential and circulatory forces), instead of the stiffness matrix. As a remarkable difference with the conservative case, when the eigenvalues $\lambda_{0}=0$ (static bifurcation) or $\lambda_{k}=i \omega_{k}$ (dynamic bifurcation) of the nonsymmetric matrix $\mathbf{F}_{\mathbf{x}}^{0}$ coalesce at a critical point, an incomplete set of eigenvectors generally exists. In this richer scenario, new interac tions appear with respect to the purely static interactions, namely, of purely dynamic (e.g. $\left.\left(i \omega_{h}, i \omega_{k}\right)\right)$ or mixed static dynamic type (e.g. $\left(0, i \omega_{k}\right)$ ). In addition, a new coupling mechanism manifest itself, said of internal resonance, which, for example, occurs when two critical frequencies, $\omega_{h}$ and $\omega_{k}$, are in an integer ratio.

All these aspects are well known in the general Bifurcation Theory, which has been developed in the framework of the Dynamical System Theory (see, e.g., $[9,10])$. Direct applications of the theory to mechanical systems have also been illustrated [11]. In this environment, the analytical tool employed is the Center Manifold Theorem in conjunction with the Normal Form Theory. The method captures the essential dynamics of the system, reducing the dimension to its linear codimension, i.e. to the sum of the algebraic multiplicities of the critical eigenvalues and of the number of the resonance conditions [12].

With rare exceptions, however, the Buckling Theory seems to have been developed independently of the general Bifurcation Theory, instead of constituting a special case of it. In spite of this, the author and his co workers, in a number of papers written in the last decade, and in accordance with some results already contained in [13], has shown that the basic ideas of the SPM can also be applied to purely dynamic and static dynamic bifurca tions $[12,1416]$. The method applied there is a version of the multiple scale method (MSM), employed in nonlinear dynamics [17], suitably suited for bifurcation problems of nondefective type (i.e. bifurcations in which a set of complete critical modes exists). Later, a new version of the MSM, valid for defective operators (i.e. with an incomplete set of eigenvectors), has also been imple mented [18 20]. More recently, the method has been extended to continuous systems [21 25]. As result of these researches, a general, unified method is available to analyse static and/or dynamic bifurcations from a known path of autonomous systems, both of discrete or continuous type. The method is alternative to the (more extensively employed) center manifold method; moreover, it is more engineering oriented and preserves all the aspects of the SPM.

The fundamental steps of the algorithm are illustrated here with reference to a divergence Hopf Hopf bifurcation $\left(0, i \omega_{h}\right.$, $i \omega_{k}$ ), of resonant or nonresonant type. Results concerning some sub cases, already studied in previous works, are resumed here. The reader is referred to an extended review [12] or to the original papers for any additional details.

\section{The multiple scale method for nondefective multiple bifurcations}

A general, $n$ dimensional autonomous system is considered, whose equations of motion read:

$\mathbf{q}=Q(\mathbf{q}, \dot{\mathbf{q}} ; \boldsymbol{\mu}), \quad \mathbf{q} \in \mathbb{R}^{n}, \quad \boldsymbol{\mu} \in \mathbb{R}^{M}$

Here, $\mathbf{q}$ is the lagrangian coordinate vector, $Q$ accounts for nonlinear forces of any type, $\boldsymbol{\mu}$ is the parameter vector and the dot denotes time differentiation. It is assumed that $Q(\mathbf{0 , 0} ; \boldsymbol{\mu})=\mathbf{0}, \forall \boldsymbol{\mu}$, i.e. the origin is an equilibrium position for any combination of the parameters (trivial path). Eq. (1) can be put in the state space form:

$\dot{\mathbf{x}}=\mathbf{F}(\mathbf{x}, \boldsymbol{\mu}), \quad \mathbf{x} \in \mathbb{R}^{N}, \quad \boldsymbol{\mu} \in \mathbb{R}^{M}, \quad \mathbf{F}(\mathbf{0}, \boldsymbol{\mu})=\mathbf{0} \forall \boldsymbol{\mu}$

where $\mathbf{x}:=(\mathbf{q}, \dot{\mathbf{q}}), \mathbf{F}:=(\dot{\mathbf{q}}, Q(\mathbf{q}, \dot{\mathbf{q}}, \boldsymbol{\mu}))$ and $N:=2 n$.

\subsection{Spectral properties of the Jacobian}

Let the Jacobian $\mathbf{F}_{\mathbf{x}}^{0}:=[\partial \mathbf{F} / \partial \mathbf{x}]_{\mathbf{x}} \quad \mathbf{0}, \boldsymbol{\mu} \quad \mathbf{0}$, evaluated at $O:=(\mathbf{x}, \boldsymbol{\mu})=(\mathbf{0}, \mathbf{0})$, admit a cluster of (critical) distinct eigenvalues $\lambda$ with zero real part, i.e. let $O$ be a bifurcation point. The remaining eigenvalues are assumed to lie on the left part of the complex plane, well separated from the imaginary axis. As an example, a specific case of divergence Hopf Hopf bifurcation is considered, for which one zero eigenvalue, $\lambda_{0}=0$, and two distinct purely imaginary eigenvalues, $\lambda_{1}=i \omega_{1}, \lambda_{2}=i \omega_{2}$, together with their complex conjugates, occur at $O$ (Fig. 2). If $r:=\omega_{2} / \omega_{1}=2,3, \ldots$, the double Hopf bifurcation is termed resonant, otherwise nonresonant.

In order to satisfy the conditions which identify the critical point, namely, $\operatorname{Re}\left[\lambda_{0}\right]=\operatorname{Re}\left[\lambda_{1}\right]=\operatorname{Re}\left[\lambda_{2}\right]=0$, plus $\operatorname{Im}\left[\lambda_{2}\right]=r \operatorname{Im}\left[\lambda_{1}\right]$, at least $M=3$ parameters are necessary in the nonresonant case, and $M=4$ parameters in the resonant case. Each of these conditions represents a surface in the parameter space. The intersection of the $M$ surfaces determines the critical manifold. Accordingly, the integer $M$ is called the (linear) codimension of the problem. 


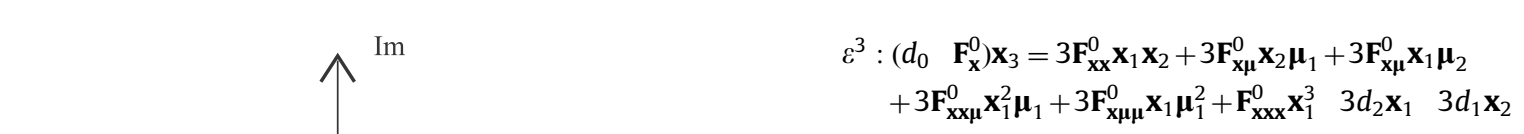

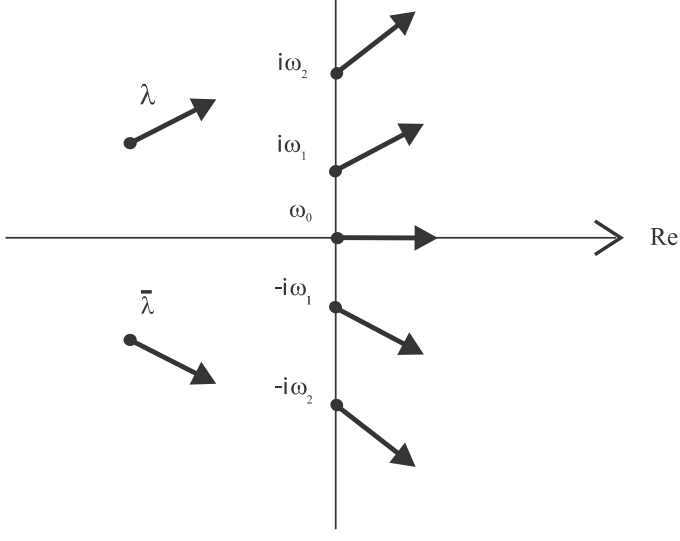

Fig. 2. Stable and critical eigenvalues at a divergence-Hopf-Hopf bifurcation point.

The (right) eigenvectors $\mathbf{u}_{0} \in \mathbb{R}$ and $\left(\mathbf{u}_{1}, \mathbf{u}_{2}\right) \in \mathbb{C}$, associated with the critical eigenvalues, are solutions of:

$\mathbf{F}_{\mathbf{x}}^{0} \mathbf{u}_{0}=\mathbf{0}, \quad \mathbf{F}_{\mathbf{x}}^{0} \mathbf{u}_{1}=i \omega_{1} \mathbf{u}_{1}, \quad \mathbf{F}_{\mathbf{x}}^{0} \mathbf{u}_{2}=i \omega_{2} \mathbf{u}_{2}$

while the left eigenvectors $\mathbf{v}_{0} \in \mathbb{R}$ and $\left(\mathbf{v}_{1}, \mathbf{v}_{2}\right) \in \mathbb{C}$ satisfy the adjoint problem:

$\left(\mathbf{F}_{\mathbf{x}}^{0}\right)^{T} \mathbf{v}_{0}=\mathbf{0}, \quad\left(\mathbf{F}_{\mathbf{x}}^{0}\right)^{T} \mathbf{v}_{1}=i \omega_{1} \mathbf{v}_{1}, \quad\left(\mathbf{F}_{\mathbf{x}}^{0}\right)^{T} \mathbf{v}_{2}=i \omega_{2} \mathbf{v}_{2}$

Right and left eigenvectors are bi orthogonal, i.e. $\mathbf{v}_{h}^{H} \mathbf{u}_{k}=\delta_{h k}$, where $\delta$ is the Kronecker symbol and the superscript $H$ denotes the transpose conjugated.

\subsection{Perturbation equations}

The equations of motion (2), after expansion around $O$, assume the form:

$\dot{\mathbf{x}}=\mathbf{F}_{\mathbf{x}}^{0} \mathbf{x}+1 / 2\left(\mathbf{F}_{\mathbf{x x}}^{0} \mathbf{x}^{2}+2 \mathbf{F}_{\mathbf{x} \mu}^{0} \mathbf{x} \boldsymbol{\mu}\right)+1 / 6\left(\mathbf{F}_{\mathbf{x x x}}^{0} \mathbf{x}^{3}+3 \mathbf{F}_{\mathbf{x x \mu}}^{0} \mathbf{x}^{2} \boldsymbol{\mu}+3 \mathbf{F}_{\mathbf{x} \mu \mu}^{0} \mathbf{x} \boldsymbol{\mu}^{2}\right)+\cdots$

where the subscript denotes $\mathbf{x}$ or $\boldsymbol{\mu}$ differentiation and the superscript 0 evaluation at the bifurcation point. Moreover, it has been utilized from Eq. (2), that $\mathbf{F}$ and its $\boldsymbol{\mu}$ derivatives vanish at the equilibrium point. Solutions of (5) are sought in parametric form $\mathbf{x}=\mathbf{x}(t, \boldsymbol{\mu}(\varepsilon)), \boldsymbol{\mu}=\boldsymbol{\mu}(\varepsilon)$, where $\ll 1$ is a perturbation para meter. By taking $\varepsilon=0$ at $O$, and expanding in series for small $\varepsilon$, it follows:

$$
\left(\begin{array}{c}
\mathbf{x}(t) \\
\boldsymbol{\mu}
\end{array}\right)=\sum_{k}^{\infty} \frac{\varepsilon^{k}}{k !}\left(\begin{array}{c}
\mathbf{x}_{k}(t) \\
\boldsymbol{\mu}_{k}
\end{array}\right)
$$

According to the MSM, several independent time scales $t_{k}$ are introduced; consequently, the time differential operator is expressed by the chain rule:

$t_{0}=t, \quad t_{1}=\varepsilon t, \quad t_{2}=\frac{\varepsilon^{2}}{2 !} t, \ldots, t_{k}=\frac{\varepsilon^{k}}{k !} t$

$\frac{d}{d t}=d_{0}+\varepsilon d_{1}+\frac{\varepsilon^{2}}{2 !} d_{2}+\cdots+\frac{\varepsilon^{k}}{k !} d_{k}+\cdots, \quad d_{k}:=\frac{\partial}{\partial t_{k}}$

By substituting Eqs. (6) and (7) in (5), and separately equating to zero terms with the same power of $\varepsilon$, the following perturba tion equations in the unknowns $\mathbf{x}_{k}$ and $\boldsymbol{\mu}_{k}$ are finally obtained:

$\varepsilon:\left(\begin{array}{ll}d_{0} & \mathbf{F}_{\mathbf{x}}^{0}\end{array}\right) \mathbf{x}_{1}=\mathbf{0}$

$\varepsilon^{2}:\left(\begin{array}{ll}d_{0} & \mathbf{F}_{\mathbf{x}}^{0}\end{array}\right) \mathbf{x}_{2}=\mathbf{F}_{\mathbf{x x}}^{0} \mathbf{x}_{1}^{2}+2 \mathbf{F}_{\mathbf{x} \boldsymbol{\mu}}^{0} \mathbf{x}_{1} \boldsymbol{\mu}_{1} \quad 2 d_{1} \mathbf{x}_{1}$

\subsection{First order solution}

The perturbation Eq. (8) are solved in chain. The $\varepsilon$ order (generating) equation admits the solution:

$\mathbf{x}_{1}=A_{0}\left(t_{1}, t_{2}, \ldots\right) \mathbf{u}_{0}+A_{1}\left(t_{1}, t_{2}, \ldots\right) \mathbf{u}_{1} e^{i \omega_{1} t_{0}}+A_{2}\left(t_{1}, t_{2}, \ldots\right) \mathbf{u}_{2} e^{i \omega_{2} t_{0}}+$ c.c.

where $A_{0} \in \mathbb{R},\left(\mathbb{A}_{1}, \mathbb{A}_{2}\right) \in \mathbb{C}$ are the slowly modulated unknown amplitudes of the critical modes, and c.c. denotes the complex conjugate of the preceding terms. It should be noted, that only motions belonging to the critical subspace $\mathcal{U}_{c}:=\left\{\mathbf{u}_{0}, \mathbf{u}_{1}, \mathbf{u}_{2}\right\}$ have been considered, namely $\mathbf{x}_{1} \in \mathcal{U}_{c}$, since they are in steady state on the $t_{0}$ scale, whereas the remaining components, being associated with stable eigenvalues, decay in time.

When $\mathbf{x}_{1}$ is substituted in the $\varepsilon^{2}$ order equation, the quadratic nonlinearities generate the 'forcing' frequencies $\left(0, \omega_{1}, \omega_{2} ; 2 \omega_{1}\right.$, $\left.2 \omega_{2}, \omega_{1}+\omega_{2}, \omega_{2} \omega_{1}\right)$, so that the equation assumes the form:

$$
\begin{aligned}
& \left(\begin{array}{ll}
d_{0} & \mathbf{F}_{\mathbf{x}}^{0}
\end{array}\right) \mathbf{x}_{2}=\left(\begin{array}{lll}
\mathbf{f}_{0} & 2 d_{1} A_{0} \mathbf{u}_{0}
\end{array}\right)+\left(\mathbf{f}_{1} \quad 2 d_{1} A_{1} \mathbf{u}_{1}\right) e^{i \omega_{1} t_{0}}+\left(\mathbf{f}_{2} \quad 2 d_{1} A_{2} \mathbf{u}_{2}\right) e^{i \omega_{2} t_{0}} \\
& +\mathbf{f}_{20} e^{2 i \omega_{1} t_{0}}+\mathbf{f}_{02} e^{2 i \omega_{2} t_{0}}+\mathbf{f}_{11} e^{i\left(\omega_{1}+\omega_{2}\right) t_{0}}+\mathbf{f}_{\overline{1} 1} e^{i\left(\omega_{2}-\omega_{1}\right) t_{0}}+\text { c.c. }
\end{aligned}
$$

where the $\mathbf{f}$ vectors are bilinear in the first order quantities $A$ 's and $\boldsymbol{\mu}_{1}$ :

$$
\begin{aligned}
& \mathbf{f}_{0}:=2 A_{0}^{2} \mathbf{F}_{\mathbf{x x}}^{0} \mathbf{u}_{0}^{2}+A_{1} \bar{A}_{1} \mathbf{F}_{\mathbf{x x}}^{0} \mathbf{u}_{1} \overline{\mathbf{u}}_{1}+A_{2} \bar{A}_{2} \mathbf{F}_{\mathbf{x x}}^{0} \mathbf{u}_{2} \overline{\mathbf{u}}_{2}+2 A_{0} \mathbf{F}_{\mathbf{x} \boldsymbol{\mu}}^{0} \mathbf{u}_{0} \boldsymbol{\mu}_{1} \\
& \mathbf{f}_{1}:=4 A_{0} A_{1} \mathbf{F}_{\mathbf{x x}}^{0} \mathbf{u}_{0} \mathbf{u}_{1}+2 A_{1} \mathbf{F}_{\mathbf{x} \boldsymbol{\mu}}^{0} \mathbf{u}_{1} \boldsymbol{\mu}_{1}, \\
& \mathbf{f}_{2}:=4 A_{0} A_{2} \mathbf{F}_{\mathbf{x x}}^{0} \mathbf{u}_{0} \mathbf{u}_{2}+2 A_{2} \mathbf{F}_{\mathbf{x} \boldsymbol{\mu}}^{0} \mathbf{u}_{2} \boldsymbol{\mu}_{1} \\
& \mathbf{f}_{20}:=A_{1}^{2} \mathbf{F}_{\mathbf{x x}}^{0} \mathbf{u}_{1}^{2}, \quad \mathbf{f}_{02}:=A_{2}^{2} \mathbf{F}_{\mathbf{x x}}^{0} \mathbf{u}_{2}^{2}, \quad \mathbf{f}_{11}:=2 A_{1} A_{2} \mathbf{F}_{\mathbf{x x}}^{0} \mathbf{u}_{1} \mathbf{u}_{2}, \\
& \mathbf{f}_{\overline{1} 1}:=2 \bar{A}_{1} A_{2} \mathbf{F}_{\mathbf{x x}}^{0} \overline{\mathbf{u}}_{1} \mathbf{u}_{2}
\end{aligned}
$$

and where an overbar denotes the complex conjugate.

Now, the forcing frequencies $\left(0, \omega_{1}, \omega_{2}\right)$ are certainly resonant, and would cause responses which diverge on the $t_{0}$ scale (secular terms). The remaining frequencies, instead, do not cause resonance if the frequency ratio $r$ assumes a generic value; in contrast, if $r=2$, then $2 \omega_{1}=\omega_{2}$ and $\omega_{2} \quad \omega_{1}=\omega_{1}$, so that $\mathbf{f}_{20}$ and $\mathbf{f}_{11}$ are also resonant. All these resonant terms must be removed from the right member, by requiring they have zero components along the critical eigenvectors $\mathbf{u}_{0}, \mathbf{u}_{1}$ and $\mathbf{u}_{2}$, respectively, i.e. they are orthogonal to the left eigenvectors $\mathbf{v}_{0}, \mathbf{v}_{1}$ and $\mathbf{v}_{2}$. By enforcing these (solvability) conditions, and accounting for bi orthogonality, one gets:

$$
d_{1} A_{0}=\frac{1}{2} \mathbf{v}_{0}^{T} \mathbf{f}_{0}, \quad d_{1} A_{1}=\frac{1}{2} \mathbf{v}_{1}^{H}\left(\mathbf{f}_{1}+\delta_{r 2} \mathbf{f}_{\overline{1} 1}\right), \quad d_{1} A_{2}=\frac{1}{2} \mathbf{v}_{2}^{H}\left(\mathbf{f}_{2}+\delta_{r 2} \mathbf{f}_{20}\right)
$$

Eq. (12) govern, for given parameters $\boldsymbol{\mu}_{1}$, the evolution of the amplitudes of the critical modes on the slow time scale $t_{1}$. They are called the (first order) amplitude modulation equations (AME). Since $A_{0}$ is real and $A_{1}, A_{2}$ are complex, Eqs. (12) are equivalent to five real equations. Therefore, the original $N$ dimensional dyna mical system (2), has been reduced to a 5 dimensional system, i.e. to the dimension of the critical subspace $\mathcal{U}_{c}$.

If the analysis is truncated at this order (first order approxima tion), then $\mathbf{x}=\varepsilon \mathbf{x}_{1}$, according to Eqs. (9) and (6). Therefore, the motion consists of two harmonic components, of frequencies $\left(\omega_{1}, \omega_{2}\right)$, slowly modulated in amplitude and phases, which occur around a buckled equilibrium state, also modulated in amplitude. The trajectories all belong to the critical subspace, namely $\mathbf{x}(t) \in \mathcal{U}_{c} \forall t$. 


\subsection{Second order solution}

If a higher order approximation for the AME is desired, the $\varepsilon^{2}$ order perturbation Eq. (10) must be solved. After using Eqs. (12) to eliminate the amplitude derivatives, the steady state solution reads:

$$
\begin{aligned}
\mathbf{x}_{2}= & 2 A_{0}^{2} \mathbf{z}_{00}+A_{1} \bar{A}_{1} \mathbf{z}_{1 \overline{1}}+A_{2} \bar{A}_{2} \mathbf{z}_{2 \overline{2}}+2 A_{0} \mathbf{z}_{0 \mu} \boldsymbol{\mu}_{1}+\left(A_{0} A_{1} \mathbf{z}_{01}+2 A_{1} \mathbf{z}_{1 \mu} \boldsymbol{\mu}_{1}\right) e^{i \omega_{1} t_{0}} \\
& +\left(4 A_{0} A_{2} \mathbf{z}_{02}+2 A_{2} \mathbf{z}_{2 \mu} \boldsymbol{\mu}_{1}\right) e^{i \omega_{2} t_{0}}+A_{1}^{2} \mathbf{z}_{11} e^{2 i \omega_{1} t_{0}}+A_{2}^{2} \mathbf{z}_{22} e^{2 i \omega_{2} t_{0}} \\
& +2 A_{1} A_{2} \mathbf{z}_{12} e^{i\left(\omega_{1}+\omega_{2}\right) t_{0}}+2 \bar{A}_{1} A_{2} \mathbf{z}_{\overline{1} 2} e^{i\left(\omega_{2}-\omega_{1}\right) t_{0}}+\text { c.c. }
\end{aligned}
$$

Here, the vectors $\mathbf{z}_{h k} \in \mathbb{C}^{N}, \mathbf{z}_{j \boldsymbol{\mu}} \in \mathbb{C}^{N} \times \mathbb{C}^{M}$ are solution of the following algebraic equations:

$$
\begin{aligned}
& {\left[i \omega_{j} \quad \mathbf{F}_{\mathbf{x}}^{0}\right] \mathbf{z}_{h k}=\mathbf{F}_{\mathbf{x x}}^{0} \mathbf{u}_{h} \mathbf{u}_{k} \quad\left(\mathbf{v}_{j}^{H} \mathbf{F}_{\mathbf{x x}}^{0} \mathbf{u}_{h} \mathbf{u}_{k}\right) \mathbf{u}_{j}, \quad j, h, k=0, \pm 1, \pm 2, \omega_{h}+\omega_{k}=\omega_{j}} \\
& {\left[\begin{array}{ll}
i \omega_{j} & \mathbf{F}_{\mathbf{x}}^{0}
\end{array}\right] \mathbf{z}_{j \boldsymbol{\mu}}=\mathbf{F}_{\mathbf{x} \boldsymbol{\mu}}^{0} \mathbf{u}_{j} \quad\left(\mathbf{v}_{j}^{H} \mathbf{F}_{\mathbf{x} \boldsymbol{\mu}}^{0} \mathbf{u}_{j}\right) \mathbf{u}_{j}, \quad j=0, \pm 1, \pm 2,} \\
& {\left[i\left(\omega_{h}+\omega_{k}\right) \quad \mathbf{F}_{\mathbf{x}}^{0}\right] \mathbf{z}_{h k}=\mathbf{F}_{\mathbf{x x}}^{0} \mathbf{u}_{h} \mathbf{u}_{k}, \quad j, h, k=0, \pm 1, \pm 2 ; \omega_{h}+\omega_{k} \neq \omega_{j}}
\end{aligned}
$$

Moreover, $\omega_{k}:=\omega_{|k|}$ and $\mathbf{u}_{k}:=\overline{\mathbf{u}}_{|k|}$ when $k<0$, and a negative subscript of $\mathbf{z}_{\alpha}$ stands for an overbarred subscript. It should be observed that, while the nonsingular Eqs. (14) $)_{3}$ admit a unique solution, the singular Eqs. (14) $)_{1,2}$, by virtue of Eqs. (3), admit $\infty^{1}$ solutions of kind $\mathbf{z}_{\alpha}=\hat{\mathbf{z}}_{\alpha}+B_{j} \mathbf{u}_{j}, \forall B_{j}$, with $\hat{\mathbf{z}}_{\alpha}$ a particular solution. However, $\mathbf{z}_{\alpha}$ can be made unique by enforcing a suitable normal ization condition, e.g. $\mathbf{v}_{j}^{H} \mathbf{z}_{\alpha}=0$; this assures that $\mathbf{x}_{2}$ does not contain the $\varepsilon$ order solution (9), i.e. it does not have harmonic components $\left(0, \omega_{1}, \omega_{2}\right)$ belonging to the critical subspace. $\mathbf{x}_{2} \notin \mathcal{U}_{c}$ describes the contribution to motion of the 'passive', stable coordinates, which also depend on the amplitudes $\left(A_{0}, A_{1}, A_{2}\right)$. Hence, the motion $\mathbf{x}=\varepsilon \mathbf{x}_{1}+(1 / 2) \varepsilon^{2} \mathbf{x}_{2}$ develops itself on a 5 dimensional manifold which is tangent to $\mathcal{U}_{c}$ (since it reduces to $\mathcal{U}_{c}$ when $\varepsilon \rightarrow 0$ ), according to the basic idea of the Center Manifold Theorem.

When $\mathbf{x}_{1}$ and $\mathbf{x}_{2}$ are substituted in the $\varepsilon^{3}$ order perturbation Eq. (8) (and use is made of Eqs. (12) to express $d_{1} \times_{2}$ ), the quadratic and cubic nonlinearities generate the 'forcing' frequen cies $\left(0, \omega_{1}, \omega_{2} ; 2 \omega_{1}, 2 \omega_{2}, \omega_{1}+\omega_{2}, \omega_{2} \omega_{1} ; 3 \omega_{1}, 3 \omega_{2}, 2 \omega_{2} \pm \omega_{1}\right.$, $\left.\omega_{2} \pm 2 \omega_{1}\right)$, so that the equation assumes the form:

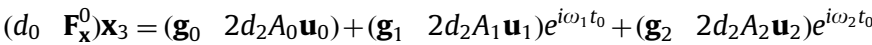

$$
\begin{aligned}
& +\mathbf{g}_{20} e^{2 i \omega_{1} t_{0}}+\mathbf{g}_{\overline{1} 1} e^{i\left(\omega_{2}-\omega_{1}\right) t_{0}} \\
& +\mathbf{g}_{30} e^{3 i \omega_{1} t_{0}}+\mathbf{g}_{\overline{2} 1} e^{i\left(\omega_{2}-2 \omega_{1}\right) t_{0}} \\
& +N R T+\text { c.c. }
\end{aligned}
$$

In this equation, only the (possibly) resonant frequencies have been displayed, while the remaining nonresonant terms (NRT) have been omitted, since they are inessential to the second order approximation. The $\mathbf{g}$ vectors in Eqs. (15) are trilinear in the first order quantities $A$ 's and $\boldsymbol{\mu}_{1}$, and/or bilinear in $A$ 's and the second order quantity $\boldsymbol{\mu}_{2}$; due to their cumbersome expressions they have been omitted here.

Eqs. (15) show that, in the resonant case $r=2$, in addition to the quadratic frequency combinations $2 \omega_{1}=\omega_{2}$ and $\omega_{2} \quad \omega_{1}=\omega_{1}$, also the cubic combination $\omega_{2} \quad 2 \omega_{1}=0$ is resonant. Moreover, a new form of cubic resonance appears in the $r=3$ case, since $3 \omega_{1}=\omega_{2}$ and $\omega_{2} 2 \omega_{1}=\omega_{1}$. Therefore, solvability conditions for Eqs. (15) lead to:

$$
\begin{aligned}
& d_{2} A_{0}=(1 / 2) \mathbf{v}_{0}^{T}\left(\mathbf{g}_{0}+\delta_{r 2} \mathbf{g}_{\overline{2} 1}\right) \\
& d_{2} A_{1}=(1 / 2) \mathbf{v}_{1}^{H}\left(\mathbf{g}_{1}+\delta_{r 2} \mathbf{g}_{\overline{1} 1}+\delta_{r 3} \mathbf{g}_{\overline{2} 1}\right) \\
& d_{2} A_{2}=(1 / 2) \mathbf{v}_{2}^{H}\left(\mathbf{g}_{2}+\delta_{r 2} \mathbf{g}_{20}+\delta_{r 3} \mathbf{g}_{30}\right)
\end{aligned}
$$

Eq. (16) are second order AME. For given parameters $\boldsymbol{\mu}_{1}$ and $\boldsymbol{\mu}_{2}$, they govern the evolution of the amplitudes $A$ 's on the slow time scale $t_{2}$. They should be solved after having determined the $t_{1}$ dependence from Eq. (12). However, since this procedure is inapplicable in the general case, an alternative method is followed. This is called reconstitution method, and consists in combining the two sets of partial differential Eqs. (12) and (16) in a unique set of ordinary differential equations, by returning to the true time $t$ [26]. Thus, by using the chain rule $(7)_{2}$, the following reconstituted AME, corrected up to second order, are obtained:

$$
\begin{aligned}
& \dot{A}_{0}=(1 / 4) \mathbf{v}_{0}^{T}\left[2 \varepsilon \mathbf{f}_{0}+\varepsilon^{2}\left(\mathbf{g}_{0}+\delta_{r 2} \mathbf{g}_{\overline{2} 1}\right)\right] \\
& \dot{A}_{1}=(1 / 4) \mathbf{v}_{1}^{H}\left[2 \varepsilon \mathbf{f}_{1}+\varepsilon^{2} \mathbf{g}_{1}+\delta_{r 2}\left(2 \varepsilon \mathbf{f}_{\overline{1} 1}+\varepsilon^{2} \mathbf{g}_{\overline{1} 1}\right)+\varepsilon^{2} \delta_{r 3} \mathbf{g}_{\overline{2} 1}\right] \\
& \dot{A}_{2}=(1 / 4) \mathbf{v}_{2}^{H}\left[2 \varepsilon \mathbf{f}_{2}+\varepsilon^{2} \mathbf{g}_{2}+\delta_{r 2}\left(2 \varepsilon \mathbf{f}_{20}+\varepsilon^{2} \mathbf{g}_{20}\right)+\varepsilon^{2} \delta_{r 3} \mathbf{g}_{30}\right]
\end{aligned}
$$

They are also referred to as bifurcation equations. Since $A_{j}=\mathrm{O}(\varepsilon), \boldsymbol{\mu}=\mathrm{O}(\varepsilon)$, and f's and g's are homogeneous of degrees 2 and 3 , respectively, in the perturbation parameter $\varepsilon$, this latter can be reabsorbed, according to the rules $\varepsilon A_{j} \rightarrow A_{j}, \varepsilon \boldsymbol{\mu}_{1}+\varepsilon^{2} \boldsymbol{\mu}_{2} \rightarrow \boldsymbol{\mu}$, $\varepsilon^{2} \boldsymbol{\mu}_{1}^{2} \rightarrow \boldsymbol{\mu}^{2}$. Finally, when the expressions for $\mathbf{f}^{\prime}$ 's and $\mathbf{g}$ 's are substituted in Eqs. (17), these assume the following form:

$$
\begin{aligned}
\dot{A}_{0}= & \mathbf{R}_{\boldsymbol{\mu} 0} \boldsymbol{\mu} A_{0}+R_{00} A_{0}^{2}+R_{1 \overline{1}} A_{1} \bar{A}_{1}+R_{2 \overline{2}} A_{2} \bar{A}_{2} \\
& +\mathbf{R}_{\boldsymbol{\mu \mu} 0} \boldsymbol{\mu}^{2} A_{0}+\mathbf{R}_{\boldsymbol{\mu} 00} \boldsymbol{\mu} A_{0}^{2}+R_{000} A_{0}^{3}+\mathbf{R}_{\boldsymbol{\mu 1} 1} \boldsymbol{\mu} A_{1} \bar{A}_{1}+\mathbf{R}_{\boldsymbol{\mu 2} \overline{2}} \boldsymbol{\mu} A_{2} \bar{A}_{2} \\
& +R_{01 \overline{1}} A_{0} A_{1} \bar{A}_{1}+R_{02 \overline{2}} A_{0} A_{2} \bar{A}_{2}+\delta_{r 2}\left(C_{\overline{11}} \bar{A}_{1}^{2} A_{2}+\bar{C}_{\overline{11} 2} A_{1}^{2} \bar{A}_{2}\right) \\
\dot{A}_{1}= & \mathbf{C}_{\boldsymbol{\mu} 1} \boldsymbol{\mu} A_{1}+C_{01} A_{0} A_{1} \\
& +\mathbf{C}_{\boldsymbol{\mu} \boldsymbol{\mu} 1} \boldsymbol{\mu}^{2} A_{1}+\mathbf{C}_{\boldsymbol{\mu} 01} \boldsymbol{\mu} A_{0} A_{1}+C_{001} A_{0}^{2} A_{1}+C_{11 \overline{1}} A_{1}^{2} \bar{A}_{1}+C_{12 \overline{2}} A_{1} A_{2} \bar{A}_{2} \\
& +\delta_{r 2} C_{\overline{1} 2} \bar{A}_{1} A_{2}+\delta_{r 3} C_{2 \overline{11}} \bar{A}_{1}^{2} A_{2} \\
\dot{A}_{2}= & \mathbf{C}_{\boldsymbol{\mu} 2} \boldsymbol{\mu} A_{2}+C_{02} A_{0} A_{2} \\
& +\mathbf{C}_{\boldsymbol{\mu} \boldsymbol{\mu} 2} \boldsymbol{\mu}^{2} A_{2}+\mathbf{C}_{\boldsymbol{\mu} 02} \boldsymbol{\mu} A_{0} A_{2}+C_{002} A_{0}^{2} A_{2}+C_{1 \overline{1} 2} A_{1} \bar{A}_{1} A_{2}+C_{22 \overline{2}} A_{2}^{2} \bar{A}_{2} \\
& +\delta_{r 2} C_{11} A_{1}^{2}+\delta_{r 3} C_{111} A_{1}^{3}
\end{aligned}
$$

where the expressions for the coefficients $R \in \mathbb{R}, \mathbf{R} \in \mathbb{R}^{\mathrm{M}}$, $C \in \mathbb{C}, \mathbf{C} \in \mathbb{C}^{\mathrm{M}}$ have been omitted here. In each of Eqs. (18), the first row contains the first order terms (quadratic), the second row the second order terms (cubic), the third row contains terms which are only present when the critical frequencies are internally resonant $(r=2,3)$. Note that the frequency associated with each term in the right hand member is equal to the frequency associated with the left hand member (e.g. $A_{1} \bar{A}_{1}$ has frequency $\omega_{1} \omega_{1}=0$ and $A_{1} A_{2} \bar{A}_{2}$ has frequency $\left.\omega_{1}+\omega_{2} \quad \omega_{2}=\omega_{1}\right)$. Moreover, the coefficients of terms with frequency 0 are real, while the others are complex. Therefore, the general structure (18) of the AME can be predicted in advance, before any calculation is performed.

\subsection{Reduced amplitude modulation equations}

To put the AME (18) in the real form, the polar representation is adopted for the complex amplitudes, namely:

$A_{0}=(1 / 2) a_{0}, \quad A_{1}=(1 / 2) a_{1} e^{i \theta_{1}}, \quad A_{2}=(1 / 2) a_{2} e^{i \theta_{2}}$

with $a_{j}$ the real amplitudes and $\theta_{j}$ the phases. When Eq. (19) are substituted in Eq. (18), and the real and imaginary parts are separately equated to zero, a set of five real equations follow. Three of them govern the amplitude modulation, namely:

$$
\begin{aligned}
\dot{a}_{0}= & \mathbf{R}_{\boldsymbol{\mu} 0} \boldsymbol{\mu} a_{0}+(1 / 2) R_{00} a_{0}^{2}+(1 / 2) R_{1 \overline{1}} a_{1}^{2}+(1 / 2) R_{2 \overline{2}} a_{2}^{2} \\
& +\mathbf{R}_{\boldsymbol{\mu} \boldsymbol{0} 0} \boldsymbol{\mu}^{2} a_{0}+(1 / 2) \mathbf{R}_{\boldsymbol{\mu} 00} \boldsymbol{\mu} a_{0}^{2}+(1 / 4) R_{000} a_{0}^{3}+(1 / 2) \mathbf{R}_{\boldsymbol{\mu 1} \overline{1}} \boldsymbol{\mu} a_{1}^{2} \\
+ & (1 / 2) \mathbf{R}_{\boldsymbol{\mu} 2 \overline{2}} \boldsymbol{\mu} a_{2}^{2}+(1 / 4) R_{01 \overline{1}} a_{0} a_{1}^{2}+(1 / 4) R_{02 \overline{2}} a_{0} a_{2}^{2} \\
+ & \delta_{r 2}(1 / 2) a_{1}^{2} a_{2}\left[R_{\overline{112}} \cos \left(2 \theta_{1} \quad \theta_{2}\right)+I_{\overline{112}} \sin \left(2 \theta_{1} \quad \theta_{2}\right)\right] \\
\dot{a}_{1}= & \mathbf{R}_{\boldsymbol{\mu} 1} \boldsymbol{\mu} a_{1}+(1 / 2) R_{01} a_{0} a_{1}+\mathbf{R}_{\boldsymbol{\mu \mu 1}} \boldsymbol{\mu}^{2} a_{1}+(1 / 2) \mathbf{R}_{\boldsymbol{\mu} 01} \boldsymbol{\mu} a_{0} a_{1} \\
& +(1 / 4) R_{001} a_{0}^{2} a_{1}+(1 / 4) R_{11 \overline{1}} a_{1}^{3}+(1 / 4) R_{12 \overline{2}} a_{1} a_{2}^{2} \\
& +\delta_{r 2}(1 / 2) a_{1} a_{2}\left[R_{\overline{12}} \cos \left(2 \theta_{1} \quad \theta_{2}\right)+I_{\overline{1} 2} \sin \left(2 \theta_{1} \quad \theta_{2}\right)\right] \\
& +\delta_{r 3}(1 / 4) a_{1}^{2} a_{2}\left[R_{2 \overline{11}} \cos \left(3 \theta_{1} \quad \theta_{2}\right)+I_{2 \overline{11}} \sin \left(3 \theta_{1} \quad \theta_{2}\right)\right]
\end{aligned}
$$




$$
\begin{aligned}
\dot{a}_{2}= & \mathbf{R}_{\boldsymbol{\mu} 2} \boldsymbol{\mu} a_{2}+\frac{1}{2} R_{02} a_{0} a_{2}+\mathbf{R}_{\boldsymbol{\mu \mu 2} 2} \boldsymbol{\mu}^{2} a_{2}+\frac{1}{2} \mathbf{R}_{\boldsymbol{\mu} 02} \boldsymbol{\mu} a_{0} a_{2} \\
& +\frac{1}{4} R_{002} a_{0}^{2} a_{2}+\frac{1}{4} R_{1 \overline{1} 2} a_{1}^{2} a_{2}+\frac{1}{4} R_{222} a_{2}^{3} \\
& +\delta_{r 2} \frac{1}{2} a_{1}^{2}\left[R_{11} \cos \left(2 \theta_{1} \quad \theta_{2}\right)+I_{11} \sin \left(2 \theta_{1} \quad \theta_{2}\right)\right] \\
& +\delta_{r 3} \frac{1}{4} a_{1}^{3}\left[R_{111} \cos \left(3 \theta_{1} \quad \theta_{2}\right)+I_{111} \sin \left(3 \theta_{1} \quad \theta_{2}\right)\right]
\end{aligned}
$$

and the remaining two the phase modulation:

$$
\begin{aligned}
& a_{1} \dot{\theta}_{1}=\mathbf{I}_{\boldsymbol{\mu} 1} \boldsymbol{\mu} a_{1}+\frac{1}{2} I_{01} a_{0} a_{1}+\frac{1}{4} \mathbf{I}_{\boldsymbol{\mu} \boldsymbol{\mu} 1} \boldsymbol{\mu}^{2} a_{1}+\frac{1}{2} \mathbf{I}_{\boldsymbol{\mu} 01} \boldsymbol{\mu} a_{0} a_{1} \\
& +\frac{1}{4} I_{001} a_{0}^{2} a_{1}+\frac{1}{4} I_{11 \overline{1}} a_{1}^{3}+\frac{1}{4} I_{12 \overline{2}} a_{1} a_{2}^{2} \\
& +\delta_{r 2} \frac{1}{2} a_{1} a_{2}\left[I_{\overline{1} 2} \cos \left(2 \theta_{1} \quad \theta_{2}\right) \quad R_{\overline{1} 2} \sin \left(2 \theta_{1} \theta_{2}\right)\right] \\
& +\delta_{r 3} \frac{1}{4} a_{1}^{2} a_{2}\left[I_{2 \overline{11}} \cos \left(3 \theta_{1} \quad \theta_{2}\right) \quad R_{2 \overline{11}} \sin \left(3 \theta_{1} \quad \theta_{2}\right)\right] \\
& a_{2} \dot{\theta}_{2}=\mathbf{I}_{\boldsymbol{\mu} 2} \boldsymbol{\mu} a_{2}+\frac{1}{2} I_{02} a_{0} a_{2}+\frac{1}{4} \mathbf{I}_{\boldsymbol{\mu \mu 2} 2} \boldsymbol{\mu}^{2} a_{2}+\frac{1}{2} \mathbf{I}_{\boldsymbol{\mu} 02} \boldsymbol{\mu} a_{0} a_{2} \\
& +\frac{1}{4} I_{002} a_{0}^{2} a_{2}+\frac{1}{4} I_{1 \overline{1} 2} a_{1}^{2} a_{2}+\frac{1}{4} I_{22 \overline{2}} a_{2}^{3} \\
& +\delta_{r 2} \frac{1}{2} a_{1}^{2}\left[I_{11} \cos \left(2 \theta_{1} \quad \theta_{2}\right) \quad R_{11} \sin \left(2 \theta_{1} \quad \theta_{2}\right)\right] \\
& +\delta_{r 3} \frac{1}{4} a_{1}^{3}\left[I_{111} \cos \left(3 \theta_{1} \quad \theta_{2}\right) \quad R_{111} \sin \left(3 \theta_{1} \quad \theta_{2}\right)\right]
\end{aligned}
$$

where $\mathbf{R}_{\alpha}:=\operatorname{Re}\left[\mathbf{C}_{\alpha}\right], \mathbf{I}_{\alpha}:=\operatorname{Im}\left[\mathbf{C}_{\alpha}\right], R_{\alpha}:=\operatorname{Re}\left[C_{\alpha}\right], I_{\alpha}:=\operatorname{Im}\left[C_{\alpha}\right]$ has been set. Important properties follow from the previous equations, as discussed below:

(a) In the nonresonant case $(r \neq 2,3, \ldots)$ the (real) amplitude equations (20) are uncoupled from the phase equations (21). Therefore, the essential dynamics is governed by a reduced set of three equations of type:

$\dot{a}_{0}=f_{0}\left(a_{0}, a_{1}, a_{2} ; \boldsymbol{\mu}\right), \quad \dot{a}_{1}=f_{1}\left(a_{0}, a_{1}, a_{2} ; \boldsymbol{\mu}\right), \quad \dot{a}_{2}=f_{2}\left(a_{0}, a_{1}, a_{2} ; \boldsymbol{\mu}\right)$

Phase modulations here play a secondary effect. They can be evaluated by the remaining Eq. (21), once the amplitude time dependence has been determined, by solving:

$a_{1} \dot{\theta}_{1}=g_{1}\left(a_{0}, a_{1}, a_{2} ; \boldsymbol{\mu}\right), \quad a_{2} \dot{\theta}_{2}=g_{2}\left(a_{0}, a_{1}, a_{2} ; \boldsymbol{\mu}\right)$

(b) In the resonant case $(r=2,3, \ldots)$ the (real) amplitude equations (20) are coupled with the phase equations (21). However, the phases do not appear individually in the amplitude equations, but just as a difference. This suggests to introduce a new variable, having the meaning of phase combination, defined as

$\gamma:=\left\{\begin{array}{lll}2 \theta_{1} & \theta_{2} & \text { if } r=2 \\ 3 \theta_{1} & \theta_{2} & \text { if } r=3\end{array}\right.$

and accordingly, to recombine the phase equations, in order to write the system in the form:

$\dot{a}_{0}=f_{0}\left(a_{0}, a_{1}, a_{2}, \gamma ; \boldsymbol{\mu}\right), \quad \dot{a}_{1}=f_{1}\left(a_{0}, a_{1}, a_{2}, \gamma ; \boldsymbol{\mu}\right)$,
$\dot{a}_{2}=f_{2}\left(a_{0}, a_{1}, a_{2}, \gamma ; \boldsymbol{\mu}\right), \quad a_{1} a_{2} \dot{\gamma}=g\left(a_{0}, a_{1}, a_{2}, \gamma ; \boldsymbol{\mu}\right)$

After having solved Eqs. (25), phases are drawn by equations of type:

$$
a_{1} \dot{\theta}_{1}=g_{1}\left(a_{0}, a_{1}, a_{2}, \gamma ; \boldsymbol{\mu}\right), \quad a_{2} \dot{\theta}_{2}=g_{2}\left(a_{0}, a_{1}, a_{2}, \gamma ; \boldsymbol{\mu}\right)
$$

By summarizing, in both cases, the essential dynamics around the bifurcation is captured by a reduced set of equations (RAME), in a number of three in the nonresonant case (a) and four in the resonant case (b), i.e., in a number equal to the codimension $M$ of the problem. This is found to be a general property, which holds for any kind of coupled instabilities.

\subsection{Steady state solutions and their stability}

The first step in analysing the RAME (22) or (25) consists in finding, may be numerically, their steady solutions $\left(a_{0 s}(\boldsymbol{\mu}), a_{1 s}(\boldsymbol{\mu}), a_{2 s}(\boldsymbol{\mu})\right)=$ const, $\gamma_{s}(\boldsymbol{\mu})=$ const (also said the fixed points of the dynamical system), as functions of the parameters. They are obtained by solving a set of $M=3,4$ nonlinear algebraic equations (steady version of RAME). The steady solutions constitute a set of paths (hyper surfaces) in the amplitude parameter space of dimension $2 M$; their plot is called the bifurcation diagram.

Since steady amplitudes and phase difference are constant, it follows from Eqs. (23) or (26), that phases vary linearly in time, $\theta_{j}=v_{j} t+\varphi_{j}(j=1,2)$, where $v_{j}$ are the frequency corrections and $\varphi_{j}$ the initial phases. No particular relations exists among these quan tities in the nonresonant case. In contrast, in the resonant case, and due to Eq. (24), the frequency corrections and initial phases are linked by $v_{2}=r v_{1}, r \varphi_{1} \quad \varphi_{2}=\gamma_{s}$, i.e. the nonlinear frequencies $\Omega_{j}:=\omega_{j}+v_{j}$ are in the same ratio $r$ as the linear frequencies $\omega_{j}$, and just one initial phase remains arbitrary, in accordance with the fact the system is autonomous (i.e. insensitive to time shifts). The steady motions, from Eqs. (9) and (19), read:

$$
\begin{aligned}
\mathbf{x}= & a_{0} \mathbf{u}_{0}+a_{1}\left(\operatorname{Re}\left[\mathbf{u}_{1}\right] \cos \left(\Omega_{1} t+\varphi_{1}\right) \quad \operatorname{Im}\left[\mathbf{u}_{1}\right] \sin \left(\Omega_{1} t+\varphi_{1}\right)\right) \\
& +a_{2}\left(\operatorname{Re}\left[\mathbf{u}_{2}\right] \cos \left(\Omega_{2} t+\varphi_{2}\right) \quad \operatorname{Im}\left[\mathbf{u}_{2}\right] \sin \left(\Omega_{2} t+\varphi_{2}\right)\right)+\text { h.o.t. }
\end{aligned}
$$

where $\varepsilon$ has been reabsorbed and higher order terms (h.o.t.) omitted. In the nonresonant case $\left(\Omega_{2} / \Omega_{1} \notin \mathbb{N}^{+}\right)$, the motion is quasi periodic (also called bi periodic); in the resonant case it is periodic $\left(\Omega_{2} / \Omega_{1}=2,3\right)$. In both cases they occur around a nontrivial buckled configuration. Eq. (27), in which amplitudes and non linear frequencies vary with $\boldsymbol{\mu}$, describe a family of tori or limit cycles, respectively, in the state space.

Stability of steady solutions is investigated by means of the variational equation, obtained by linearizing the RAME around their fixed points, namely:

$\delta \dot{\mathbf{a}}=\mathbf{f}_{\mathbf{a}}^{s} \delta \mathbf{a}$ or $\quad\left(\begin{array}{c}\delta \dot{\mathbf{a}} \\ \delta \dot{\gamma}\end{array}\right)=\left(\begin{array}{ll}\mathbf{f}_{\mathbf{a}}^{s} & \mathbf{f}_{\gamma}^{s} \\ g_{\mathbf{a}}^{s} & g_{\gamma}^{s}\end{array}\right)\left(\begin{array}{c}\delta \mathbf{a} \\ \delta \gamma\end{array}\right)$

where $\mathbf{a}:=\left(a_{0}, a_{1}, a_{2}\right)^{T}, \mathbf{f}:=\left(f_{0}, f_{1}, f_{2}\right)^{T}$, the subscript denotes differentiation with respect the homonymous variable, and the superscript $s$ evaluation at the $\boldsymbol{\mu}$ depending steady values. (It should be noted that, if $a_{1 s}$ and/or $a_{2 s}$ vanish, Eqs. (28) $)_{2}$ cannot be put in the standard form see Eq $(25)_{4}$; in this case, use must be made of the Cartesian form to express complex quantities [27]). The eigenvalues of the $M \times M$ Jacobian matrix govern the stability of the steady solutions. If, for example, at some value of $\boldsymbol{\mu}$, a zero eigenvalue is encountered, an amplitude divergence occurs, denoting branching of a new harmonic steady motion. If, for example, a purely imaginary eigenvalue is encountered, an amplitude Hopf bifurcation occurs (also called secondary Hopf bifurcation), denoting the branching of a new harmonic, periodi cally modulated motion (quasi periodic motion). The MSM can, of course, be again applied to the RAME, in order to analyse these secondary bifurcations.

\section{Examples: divergence-Hopf and double Hopf bifurcations}

Using the results achieved in Section 2, three examples (taken from [14 16]) are briefly illustrated, in which some sample 

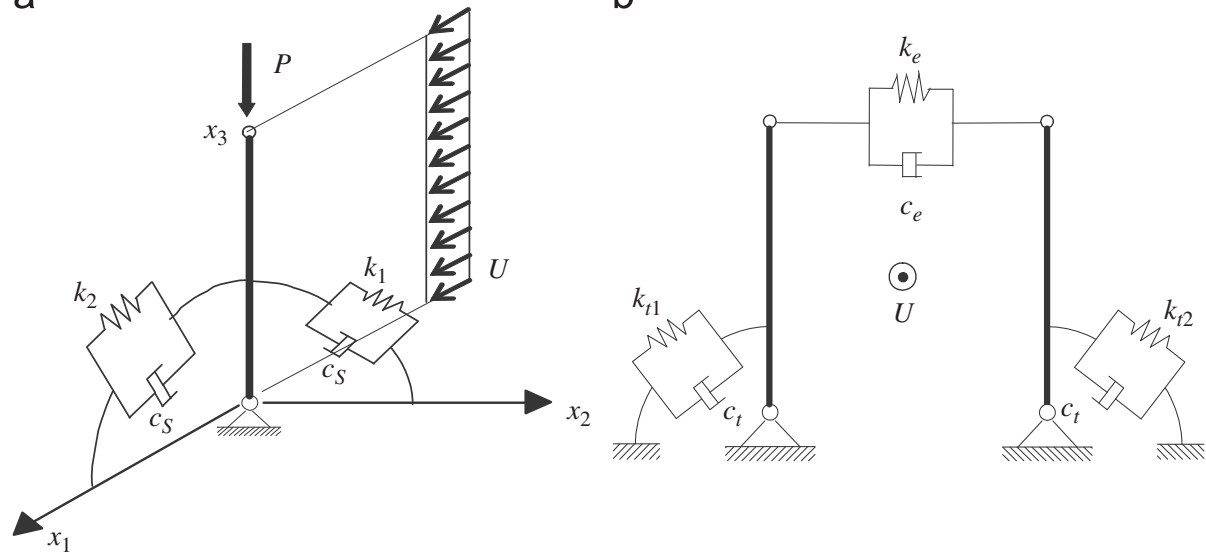

Fig. 3. Structures undergoing: (a) divergence-Hopf bifurcation and (b) Hopf-Hopf bifurcation, of nonresonant or resonant type.

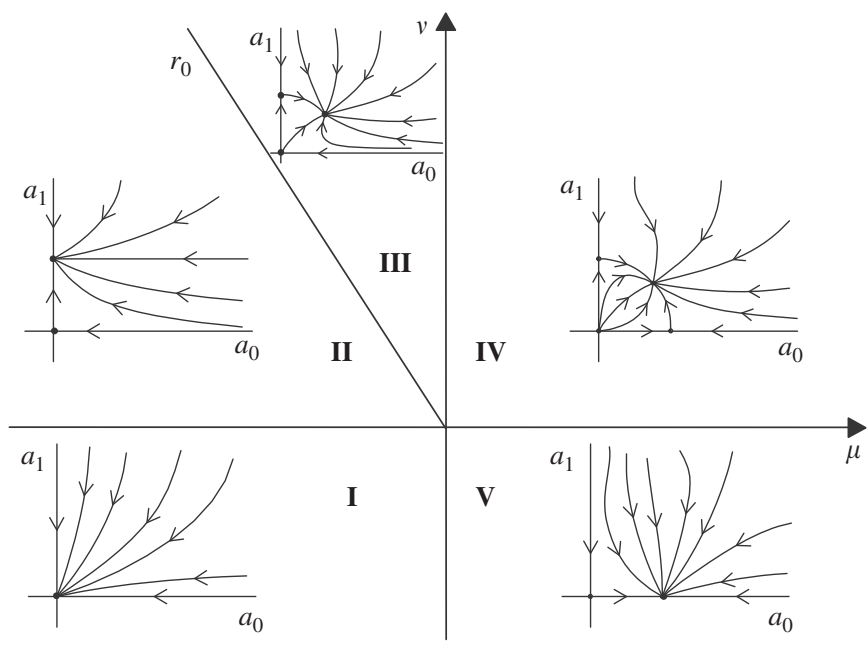

Fig. 4. Bifurcation map for the structure of Fig. 3a, undergoing divergence-Hopf bifurcation; $\mu$ load parameter, and $v$ wind parameter.

structures undergo, (a) divergence Hopf, (b) nonresonant Hopf Hopf and, (c) resonant Hopf Hopf bifurcations, respectively. The structures considered are illustrated in Fig. 3.

\subsection{Divergence Hopf bifurcation}

The structure shown in Fig. 3a consists of a rigid rod, with symmetric cross section with respect the $x_{1}$ axis, spherically hinged, elastically restrained and damped at ground, subjected to a dead load $P$ and a wind flow $U$. The dead load is responsible, at $P=P_{c}$, for buckling in the $\left(x_{1}, x_{3}\right)$ plane, of minor stiffness; the wind flow, at $U=U_{c}$, triggers a Hopf bifurcation in the $\left(x_{2}, x_{3}\right)$ plane. A static dynamic instability interaction takes place around the point $C:=$ $\left(U_{c}, P_{c}\right)$ of the parameter plane (codimension $\left.M=2\right)$. Drag forces, acting in the buckling plane, are neglected, in order the system admits the trivial solution. A more refined analysis, accounting for these latter as imperfections, has been carried out in [15].

The dynamics around $C$ is governed by the following amplitude equations [15], which are a particular case of Eqs. (22):

$\dot{a}_{0}=\frac{1}{2 \xi_{s}}\left(\mu a_{0} \quad \frac{1}{6} a_{0}^{3}+\frac{1}{4} a_{0} a_{1}^{2}\right), \quad \dot{a}_{1}=\frac{1}{2} \xi_{a} v a_{1}+\frac{3}{8} \frac{c_{3}}{u_{c}} \omega^{2} a_{1}^{3}$

in which $a_{0}$ is the buckling amplitude, $a_{1}$ the oscillation amplitude, $\omega$ its linear frequency, $\xi_{s}$ and $\xi_{a}$ the structural and

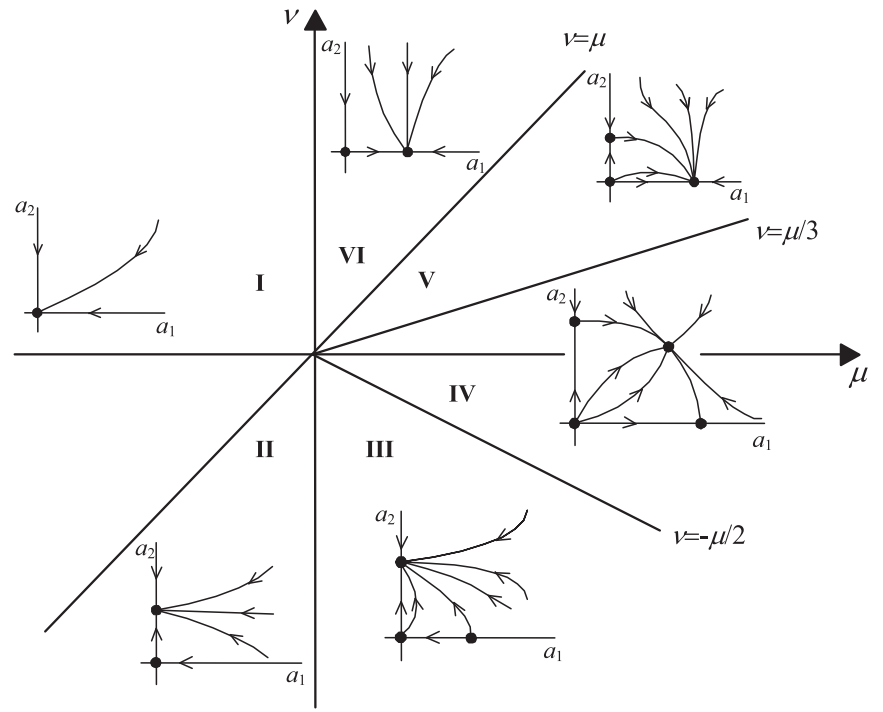

Fig. 5. Bifurcation map for the structure of Fig. 3b, undergoing nonresonant HopfHopf bifurcation; $\mu$ wind parameter, and $v$ damping parameter.

aerodynamic damping coefficients, respectively, $c_{3}<0$ a nonlinear aerodynamic coefficient, $u_{c}$ a nondimensional wind velocity, $\mu$ the increment of nondimensional load and $v$ the increment of the nondimensional wind, measured from their critical values. Analysis of Eqs. (29) leads to the bifurcation chart of Fig. 4. Here, the parameter plane has been divided in regions in which the system has qualitatively similar behaviours, sketched by phase portraits. In region I the trivial equilibrium is stable; in region II a stable periodic motion around the trivial equilibrium takes place; in regions III and IV the periodic motion occurs around the buckled configuration; in region $\mathrm{V}$ only buckling occurs. It should be noticed that, the interaction triggers buckling even in the (otherwise stable) region III.

\subsection{Nonresonant Hopf Hopf bifurcation}

The structure shown in Fig. 3b consists of two planar rigid rods, elastically restrained and damped at the ground, connected at the free ends by a visco elastic extensional device (e.g. an active control device, able to furnish forces of both signs). The system is loaded by a wind flow $U$ blowing orthogonally to the plane of the structure, causing in plane instability of a purely vibrational type. Both rods, considered independently, experience Hopf bifurcation; due to the 
coupling device, they interact. A symmetric structure is considered here $\left(k_{t 1}=k_{t 2}\right)$ for which oscillations manifest in an antisymmetric mode (rods rotating in phase), of amplitude $a_{1}$, and in a symmetric mode (rods rotating opposite in phase), of amplitude $a_{2}$. For damping $c_{e}$ of the coupling device equal to zero, the two Hopf bifurcations occur simultaneously at a certain wind velocity $U_{c}$. Interaction then occurs around the point $C:=\left(U_{c}, 0\right)$ of the $\left(U, c_{e}\right)$ parameter plane (codimension $M=2$ bifurcation). Here, dynamics is governed by the following amplitude equations [14], which are a particular case of Eqs. (22):

$a_{1} \quad \frac{1}{2} \xi_{a} \mu a_{1}+\frac{1}{4} \frac{c_{3}}{u_{c}} \omega^{2} a_{1} a_{2}^{2}+\frac{3}{8} \frac{c_{3}}{u_{c}} a_{1}^{3}, \quad a_{2} \quad \frac{1}{2} \xi_{a}(\mu \quad v) a_{2}+\frac{1}{4} \frac{c_{3}}{u_{c}} a_{1}^{2} a_{2}+\frac{3}{8} \frac{c_{3}}{u_{c}} \omega^{2} a_{2}^{3}$

In these, $\xi_{a}, c_{3}, u_{c}$ and $\omega$ keep the meaning previously assumed, $\mu$ is the nondimensional incremental wind velocity and $v$ the (positive or negative) nondimensional damping of the coupling device. Analysis of Eqs. (30) leads to the bifurcation map of Fig. 5.
In region I the trivial solution is stable. In regions II and VI, a stable symmetric or antisymmetric periodic motion (limit cycle) takes place, respectively. These persist also in regions III and V, after the appearance of an unstable mode of opposite type. Finally, in region $\mathrm{IV}$, both monomodal solutions lose stability, and a stable bimodal, quasi periodic motion exists.

\subsection{Resonant Hopf Hopf bifurcation}

The same structure as in Fig. 3b is considered, but asymmetric, with $k_{t 1} \neq k_{t 2}$. In order two Hopf bifurcations occur simulta neously, having critical frequencies in an integer ratio, three parameters are necessary, namely $\boldsymbol{\mu}=(\mu, v, \rho)$. These are chosen as the (incremental) nondimensional wind velocity $\mu$, the nondi mensional coupling stiffness $v$ and the detuning $\rho:=\left(k_{t 2} / k_{t 1}\right)\left(k_{t 2} /\right.$ $\left.k_{t 1}\right)_{c}$ between the actual and the critical value of the torsional stiffness ratio. The equations governing the dynamics around the codimension 3 point $C:=(\mu, v, \rho)=(0,0,0)$ have been obtained

a

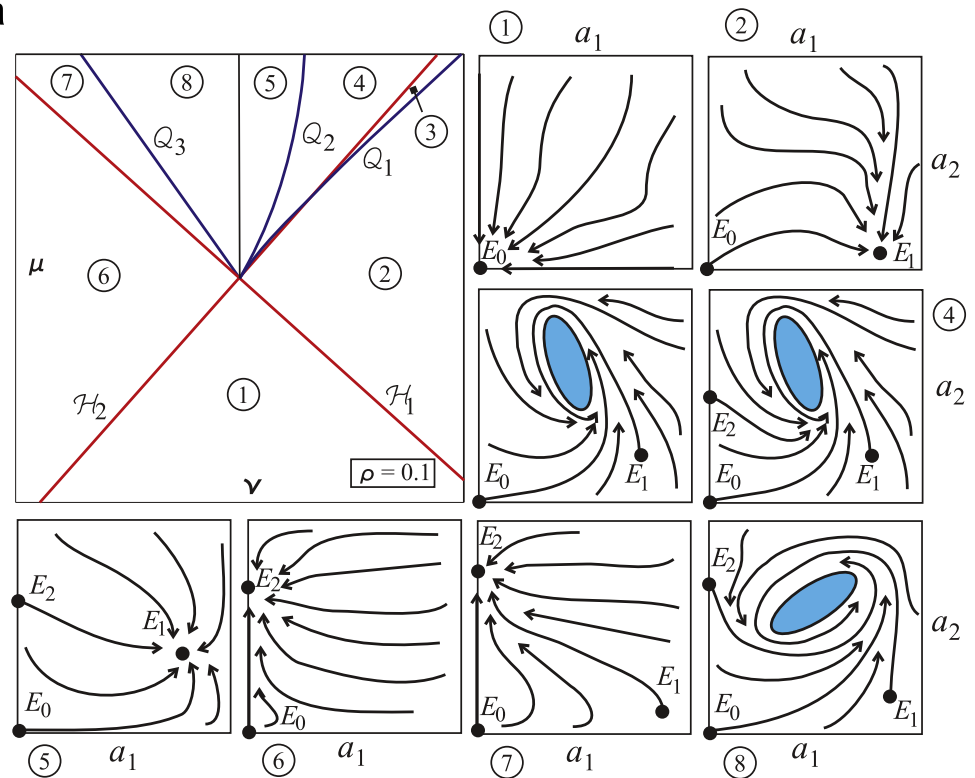

b

(1) $a_{1}$
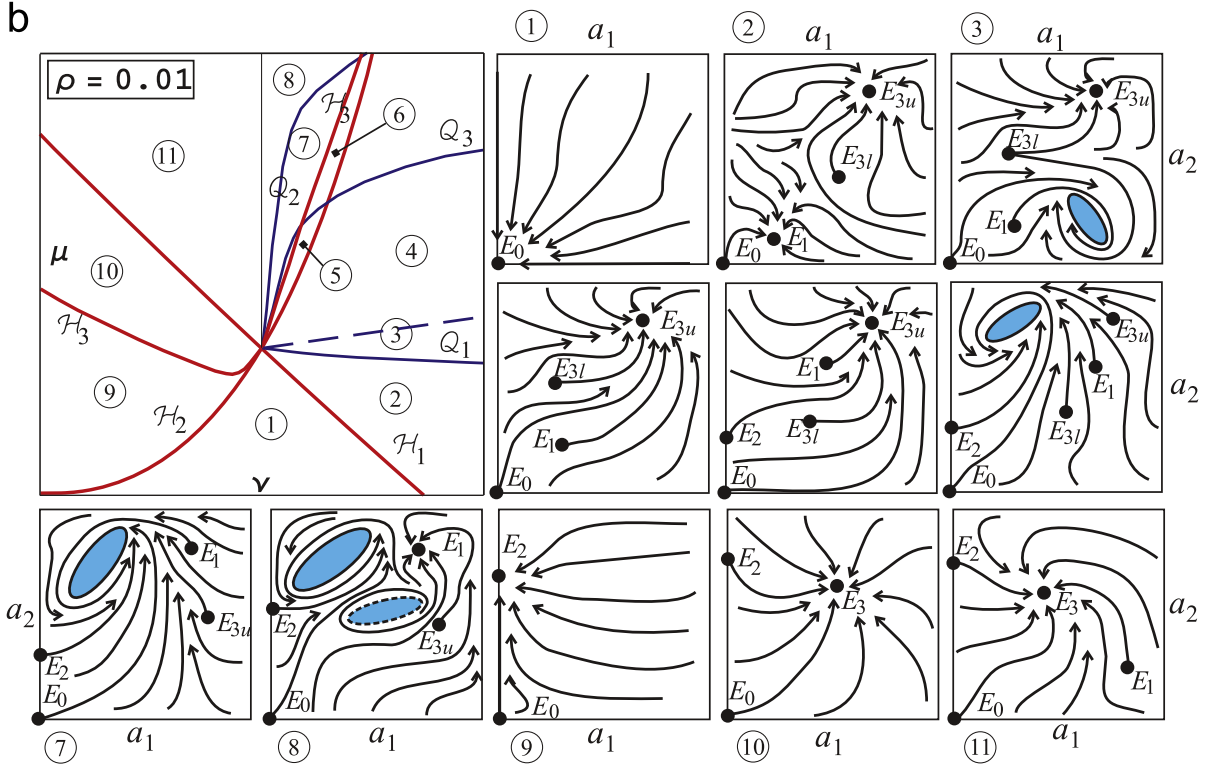

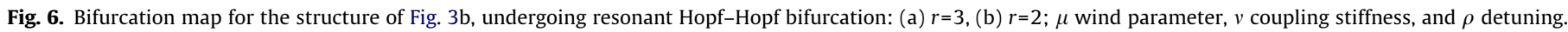


in [16], and are a particular case of Eqs. (25):

$$
\begin{aligned}
& \dot{a}_{1}=c_{1}(\boldsymbol{\mu}) a_{1}+c_{2} a_{1}^{3}+c_{3} a_{1} a_{2}^{2}+\delta_{r 2} a_{1} a_{2}\left(c_{4} \cos \gamma+c_{5} \sin \gamma\right) \\
& \quad+\delta_{r 3} a_{1}^{2} a_{2}\left(c_{6} \cos \gamma+c_{8} \sin \gamma\right) \\
& \dot{a}_{2}=c_{8}(\boldsymbol{\mu}) a_{2}+c_{9} a_{1}^{2} a_{2}+c_{10} a_{2}^{3}+\delta_{r 2} a_{1}^{2}\left(c_{11} \cos \gamma+c_{12} \sin \gamma\right) \\
& \quad+\delta_{r 3} a_{1}^{3}\left(c_{13} \cos \gamma+c_{14} \sin \gamma\right) \\
& a_{1} a_{2} \dot{\gamma}=c_{15}(\boldsymbol{\mu}) a_{1} a_{2}+c_{16} a_{1}^{3} a_{2}+c_{17} a_{1} a_{2}^{3} \\
& \quad+\delta_{r 2}\left[a_{1} a_{2}^{2}\left(c_{18} \cos \gamma-c_{19} \sin \gamma\right)+a_{1}^{3}\left(c_{20} \cos \gamma-c_{21} \sin \gamma\right)\right] \\
& \quad+\delta_{r 3}\left[a_{1}^{2} a_{2}^{2}\left(c_{22} \cos \gamma-c_{23} \sin \gamma\right)+a_{1}^{4}\left(c_{24} \cos \gamma-c_{25} \sin \gamma\right)\right]
\end{aligned}
$$

where $a_{1}$ and $a_{2}$ are the amplitudes of the two (nonsymmetric) interacting modes, $\gamma$ is the phase-difference, assuming one of the alternative expressions given in Eq. (24), and $c_{i}$ are coefficients depending on system parameters, not reported here.

A numerical analysis of Eqs. (31) furnishes the results displayed in Figs. 6a and b, in the $r=3,2$ cases, respectively. Only a section of the three-dimensional bifurcation chart is shown, at a fixed value of the detuning parameter $\rho$. Moreover, qualitative sketches of the trajectories belonging to the $\left\{a_{1}, a_{2}, \gamma\right\}$-space have been projected onto the $\left(a_{1}, a_{2}\right)$-plane. A very rich scenario is found, organized by $H$-curves, at which amplitude-divergence bifurcations occur (i.e. Hopf bifurcations for the original system) and by Q-curves, at which amplitude-Hopf bifurcations manifest themselves (i.e. secondary Hopf for the original system, leading to quasiperiodic motions). In the phase-portraits, points $E_{i}$ and cycles denote the fixed points and the periodic orbits of Eq. (31), respectively (i.e. periodic or quasi-periodic motions of the structure). Homoclinic bifurcations also occur on the positive $\mu$-half-axis. As an important result, while in the $r=3$ case just one attractor is found to exist in each region (Fig. 6a), in the $r=2$ case two attractors are in competition in some regions, so that the initial conditions decide the regime motion (Fig. $6 b$ ).

\section{Conclusions and remarks}

A general perturbation algorithm, based on the multiple scale method, has been illustrated, able to analyse static/dynamic multiple bifurcations of multiparameter autonomous systems. The method develops through the same steps of the static perturbation method, namely: (1) expand by series both state-variables and parameters; (2) obtain the perturbation equations; (3) solve in sequence these equations, and enforce solvability conditions at any step; (4) collect all the solvability conditions and obtain the bifurcation equations. As peculiar aspects of the dynamical problem, however: (a) the amplitudes depend on time; (b) several time scales are introduced; (c) the solvability and bifurcation equations are differential, instead of algebraic, equations. As peculiar aspects of nonconservative systems, two sets, of right and left eigenvectors, must be considered. Moreover, as distinct from the standard analysis carried out in elastic buckling, more parameters (not just the load) are considered simultaneously, in order to exhaustively describe the system behaviour around the critical point.

Only the simplest case of complete set of critical eigenvectors has been discussed here. When, instead, two or more eigenvalues coalesce at the critical point, generic systems do not posses a complete set of proper eigenvectors (they have not-diagonal, Jordan-block Jacobian matrices). In these cases, as known from Algebra, generalized eigenvectors must be introduced. Moreover, the perturbation algorithm must be modified by using fractional (instead of integer) power expansions and time scales [18,20].

The multiple scale method also works for coupled bifurcations of continuous systems, both of nondefective or defective type (see e.g. $[23,25])$. In these cases, care must be taken in deriving the adjoint differential operator and boundary conditions, in order to obtain the left eigenvectors. The bilinear (or Green) identity is the tool to enforce solvability at each step of the procedure.

\section{Acknowledgement} 9019.

This research was supported by INTAS Grant no. 06-1000013-

\section{References}

[1] Supple WJ. Coupled branching configurations in the elastic buckling of symmetric structural system. International Journal of Mechanical Science 1967;9:97-112.

[2] Budiansky B. Theory of buckling and post-buckling behaviour of elastic structures. In: Chia-Shun Yih, editor. Advances in applied mechanics. New York: Academic Press; 1974. p. 14.

[3] Gioncu V. General theory of coupled instabilities. Thin-Walled Structures 1994;19:81-127.

[4] Rondal J, Dubina D, Gioncu V, editors. Coupled instabilities in metal structures. In: Proceedings of the CIMS'94, Thin-Walled Struct [special issue], 1994. p. 19-20.

[5] Rondal J, Dubina D, Gioncu V, editors. Coupled instabilities in metal structures. In: Proceedings of the CIMS'96. London: Imperial College Press; 1996.

[6] Sewell MJ. The static perturbation technique in buckling problems. Journal of the Mechanics and Physics of Solids 1965;13.

[7] Thompson JMT, Hunt GW. A general theory of elastic stability. London: Wiley \& Sons; 1973.

[8] Pignataro M, Rizzi N, Luongo A. Bifurcation, stability and postcritical behaviour of elastic structures. Amsterdam: Elsevier Science Publishers; 1990.

[9] Arnold VI. Geometrical methods in the theory of ordinary differential equations. New York, Heidelberg, Berlin: Springer-Verlag; 1982. [Russian original, Moscow, 1977].

[10] Guckenheimer J, Holmes P. Nonlinear oscillations, dynamical systems and bifurcations of vector fields. New York: Springer-Verlag; 1983.

[11] Troger H, Steindl A. Nonlinear stability and bifurcation theory. Wien, New York: Springer-Verlag; 1991.

[12] Luongo A, Di Egidio A, Paolone A. Multiple scale bifurcation analysis for finitedimensional autonomous systems. In: Recent research developments in sound \& vibration, Kerala, India: Transworld Research Network; 2002. p. 161-201 [ISBN: 81-7895-031-6].

[13] Iooss G, Joseph DD. Elementary stability and bifurcation theory. New York: Springer-Verlag; 1980

[14] Luongo A, Paolone A. Perturbation methods for bifurcation analysis from multiple nonresonant complex eigenvalues. Nonlinear Dynamics 1997:14:193-210.

[15] Luongo A, Paolone A. Multiple scale analysis for divergence-Hopf bifurcation of imperfect symmetric systems. Journal of Sound and Vibration 1998;218:527-39.

[16] Luongo A, Paolone A, Di Egidio A. multiple time scales analysis for 1:2 and 1:3 resonant Hopf bifurcations. Nonlinear Dynamics 2003;34:269-91.

[17] Nayfeh AH. Perturbation methods. New York: John Wiley \& Sons; 1973.

[18] Luongo A, Di Egidio A, Paolone A. Multiple time scale analysis for bifurcation from a multiple-zero eigenvalue. AIAA Journal 2003;41(6):1143-50.

[19] Gattulli V, Di Fabio F, Luongo A. One to one resonant double Hopf bifurcation in aeroelastic oscillators with tuned mass dampers. Journal of Sound and Vibration 2003;262(2):201-17.

[20] Luongo A, Di Egidio A, Paolone A. Multiscale analysis of defective multipleHopf bifurcations. Computers \& Structures 2004;82(31-32):2705-22.

[21] Luongo A, Di Egidio A. Bifurcation equations through multiple-scales analysis for a continuous model of a planar beam. Nonlinear Dynamics 2005;41: 171-90.

[22] Paolone A, Vasta M, Luongo A. Flexural-torsional bifurcations of a cantilever beam under potential and circulatory forces: part I. Nonlinear model and stability analysis. International Journal of Nonlinear Mechanics 2006;41(4):586-94.

[23] Paolone A, Vasta M, Luongo A. Flexural-torsional bifurcations of a cantilever beam under potential and circulatory forces: Part II. Post-critical analysis. International Journal of Nonlinear Mechanics 2006;41(4):595-604.

[24] Luongo A, Di Egidio A. Divergence, Hopf and double-zero bifurcations of a nonlinear planar beam. Computers \& Structures 2006;84:1596-605.

[25] Di Egidio A, Luongo A, Paolone A. Linear and nonlinear interactions between static and dynamic bifurcations of damped planar beams. International Journal of Nonlinear Mechanics 2007;42:88-98.

[26] Luongo A, Paolone A. On the reconstitution problem in the multiple time scale method. Nonlinear Dynamics 1999;14:133-56.

[27] Luongo A, Di Egidio A, Paolone A. Computational problems in multiple scale analysis. In: Recent research developments in sound \& vibration. Kerala, India: Transworld Research Network; 2003. p. 1-31 [ISBN: 81-7736-186-4]. 